\title{
Effects of Electromagnetic Field on the Dynamical Instability of Cylindrical Collapse
}

\author{
M. Sharif *and M. Azam ${ }^{\dagger}$ \\ Department of Mathematics, University of the Punjab, \\ Quaid-e-Azam Campus, Lahore-54590, Pakistan.
}

\begin{abstract}
The objective of this paper is to discuss the dynamical instability in the context of Newtonian and post Newtonian regimes. For this purpose, we consider non-viscous heat conducting charged isotropic fluid as a collapsing matter with cylindrical symmetry. Darmois junction conditions are formulated. The perturbation scheme is applied to investigate the influence of dissipation and electromagnetic field on the dynamical instability. We conclude that the adiabatic index $\Gamma$ has smaller value for such a fluid in cylindrically symmetric than isotropic sphere.
\end{abstract}

Keywords: Collapse equation; Instability; Electromagnetic field.

PACS: 04.20.-q; 04.40.-b; 04.40.Dg; 04.40.Nr.

\section{Introduction}

The stability problem of relativistic stars has been an interesting subject for researchers. The static stellar model would be interesting if it remains stable

*msharif.math@pu.edu.pk

†azammath@gmail.com 
under fluctuations. This can be well discussed in terms of adiabatic index $\Gamma$. The dynamical stability [1]-4] of isotropic spheres in general is given by

$$
\Gamma \geq \frac{4}{3}+n \frac{M}{R}
$$

where $n$ is a number of order unity that depends on the structure of the star and $M$ and $R$ are the mass and radius of the star respectively. For example, for white dwarfs $n=2.25$.

Different physical aspects of fluids play a key role in the dynamical instability and evolution of self-gravitating systems. It was shown [5] that the dissipation increases the instability range at Newtonian corrections but makes the fluid less unstable at relativistic corrections. Chan et al. [6] found that anisotropy and radiation affect the instability range at Newtonian and post Newtonian $(\mathrm{pN})$ regimes. The same authors [7] explored that the shearing viscosity decreases the instability range of the fluid both at Newtonian and $\mathrm{pN}$ regimes. Chan [8] studied collapsing radiating star with shear viscosity and concluded that shear viscosity would increase anisotropy of pressure as well as effective adiabatic index. Dev and Gleiser [9] investigated the dynamical instability of neutral fluid sphere with varying energy density and showed that anisotropy enhances the stability whenever the tangential pressure is greater than the radial pressure. Sharif and Kausar [10] discussed the dynamical instability of expansionfree fluid at Newtonian and $\mathrm{pN}$ order in $f(R)$ gravity.

In a strong gravitational field, a star requires more charge to be in stable form. The effect of electric charge in a self-gravitating bound system has been studied by Rosseland [11]. Bekenstein [12] found that charge density has a significant effect on the system but unable to show whether the star is stable or not. Glazer [13] investigated the effect of electric charge upon the dynamical instability of isotropic fluid. Zhang et al. [14] showed that the electric charge changes the structure of neutron star when the charge and mass density are same. Further, the presence of electric field increases the stability of the star [15].

Esculpi and Aloma [16] found that both anisotropy and electric charge would boost the stability of sphere under radial adiabatic perturbation as compared to isotropic distribution of matter. Ernesto and Simeone [17] investigated that the inclusion of charge extends the stability regions for both bubbles and shells around a black hole. Joshi and Malafarina [18] studied the stability of Oppenheimer, Snyder and Datt black holes under small tangential 
pressure. They found that this black hole is not stable within the collapsing cloud whose final fate of collapse is a naked singularity rather than a black hole. In a recent paper [19], we have discussed the effects of electromagnetic field on the dynamical instability of a spherically symmetric expansionfree gravitational collapse.

Some recent work [20]-23] indicate great interest in cylindrical gravitational collapse with different fluids with and without electromagnetic field. In this paper, we use cylindrically symmetric distribution of collapsing isotropic fluid to discuss the dynamical instability with electromagnetic field. Darmois junction conditions [24] are used to match the interior non-rotating cylindrically symmetric spacetime to exterior cylindrically symmetric spacetime in the retarded time coordinates. The paper is organized as follows. In section 2, we formulate the Einstein-Maxwell field equations. We discuss junction conditions in section 3 . In section 4 , the perturbation scheme is applied on the field as well on the dynamical equations. Section 5 provides the discussion of the dynamical instability in terms of non-dissipative and dissipative perturbation. We conclude the results in the last section.

\section{Interior Fluid Distribution and the Field Equations}

We consider a collapsing cylinder bounded by a hypersurface $\Sigma$. The line element for the interior region has the following form

$$
d s_{-}^{2}=-A^{2} d t^{2}+B^{2} d r^{2}+C^{2} d \theta^{2}+d z^{2},
$$

where $A, B$, and $C$ are functions of $t$ and $r$. To preserve the cylindrical symmetry, we have the following constraints on the coordinates

$$
-\infty \leq t \leq \infty, \quad 0 \leq r<\infty, \quad-\infty<z<\infty, \quad 0 \leq \theta \leq 2 \pi,
$$

where we assume comoving coordinates inside the hypersurface. The interior coordinates are taken as $\left\{\chi^{\alpha}\right\}=\{t, r, \theta, z\}(\alpha=0,1,2,3)$. The source in the field equations is assumed to be locally dissipative in terms of heat flow given as [5], 25]

$$
T_{\alpha \beta}^{-}=(\mu+p) u_{\alpha} u_{\beta}+p g_{\alpha \beta}+q_{\alpha} u_{\beta}+q_{\beta} u_{\alpha},
$$

where $\mu$ is the energy density, $p$ is the isotropic pressure, $u_{\alpha}$ and $q_{\alpha}$ are four-velocity and radial heat flux vector of the fluid respectively satisfying 
$q_{\alpha} u^{\alpha}=0$. Moreover, we have

$$
u^{\alpha}=A^{-1} \delta_{0}^{\alpha}, \quad q^{\alpha}=q \delta_{1}^{\alpha}, \quad u^{\alpha} u_{\alpha}=-1 .
$$

The Maxwell equations can be written as

$$
F_{\alpha \beta}=\phi_{\beta, \alpha}-\phi_{\alpha, \beta}, \quad F_{; \beta}^{\alpha \beta}=4 \pi J^{\alpha},
$$

where $\phi_{\alpha}$ is the four potential and $J^{\alpha}$ is the four current. The electromagnetic energy-momentum tensor is given by

$$
E_{\alpha \beta}=\frac{1}{4 \pi}\left(F_{\alpha}^{\gamma} F_{\beta \gamma}-\frac{1}{4} F^{\gamma \delta} F_{\gamma \delta} g_{\alpha \beta}\right)
$$

where $F_{\alpha \beta}$ is the Maxwell field tensor. Since the charge per unit length of the cylinder is at rest with respect to comoving coordinates, the magnetic field will be zero in this local coordinate system [23], [26]. Thus we can write

$$
\phi_{\alpha}=(\phi(t, r), 0,0,0), \quad J^{\alpha}=\zeta u^{\alpha},
$$

where $\phi(t, r)$ and $\zeta$ is the scalar potential and charge density. The conservation of charge requires

$$
s(r)=4 \pi \int_{0}^{r} \zeta B C d r
$$

which is the electric charge interior to radius $r$. Using Eq.(1), the Maxwell equations (4) yield

$$
\begin{aligned}
\phi^{\prime \prime}-\left(\frac{A^{\prime}}{A}+\frac{B^{\prime}}{B}-\frac{C^{\prime}}{C}\right) \phi^{\prime} & =4 \pi \zeta A B^{2}, \\
\dot{\phi}^{\prime}-\left(\frac{\dot{A}}{A}+\frac{\dot{B}}{B}-\frac{\dot{C}}{C}\right) \phi^{\prime} & =0 .
\end{aligned}
$$

Here dot and prime represent derivatives with respect to $t$ and $r$ respectively. Integration of Eq.(8) with respect to $r$, assuming $\phi^{\prime}(t, 0)=0$, gives

$$
\phi^{\prime}=\frac{A B}{C} s(r) .
$$

The electric field intensity is defined as

$$
E(t, r)=\frac{s(r)}{2 \pi C} .
$$


The Einstein-Maxwell field equations, $G_{\alpha \beta}^{-}=8 \pi\left(T_{\alpha \beta}^{-}+E_{\alpha \beta}^{-}\right)$, for the interior metric gives

$$
\begin{aligned}
4 \pi A^{2}\left(2 \mu+\pi E^{2}\right) & =\left(\frac{A}{B}\right)^{2}\left(\frac{B^{\prime} C^{\prime}}{B C}-\frac{C^{\prime \prime}}{C}\right)+\frac{\dot{B} \dot{C}}{B C} \\
8 \pi A B^{2} q & =\frac{\dot{C}^{\prime}}{C}-\frac{\dot{C} A^{\prime}}{C A}-\frac{\dot{B} C^{\prime}}{B C} \\
4 \pi B^{2}\left(2 p-\pi E^{2}\right) & =\left(\frac{B}{A}\right)^{2}\left(\frac{\dot{A} \dot{C}}{A C}-\frac{\ddot{C}}{C}\right)+\frac{A^{\prime} C^{\prime}}{A C} \\
4 \pi\left(2 p+\pi E^{2}\right) & =\left(\frac{1}{A B}\right)\left(\frac{A^{\prime \prime}}{B}-\frac{\ddot{B}}{A}+\frac{\dot{A} \dot{B}}{A^{2}}-\frac{A^{\prime} B^{\prime}}{B^{2}}\right) \\
4 \pi\left(2 p+\pi E^{2}\right) & =\frac{A^{\prime \prime}}{A B^{2}}-\frac{\ddot{B}}{A^{2} B}+\frac{\dot{A} \dot{B}}{A^{3} B}-\frac{A^{\prime} B^{\prime}}{A B^{3}}+\frac{\dot{A} \dot{C}}{A^{3} C}-\frac{\ddot{C}}{A^{2} C} \\
& -\frac{B^{\prime} C^{\prime}}{B^{3} C}+\frac{C^{\prime \prime}}{B^{2} C}+\frac{A^{\prime} C^{\prime}}{A B^{2} C}-\frac{\dot{B} \dot{C}}{A^{2} B C} .
\end{aligned}
$$

Equations (15) and (16) yield

$$
\frac{1}{A^{2}}\left(\frac{\dot{A} \dot{C}}{A C}-\frac{\dot{B} \dot{C}}{B C}\right)+\frac{1}{B^{2}}\left(\frac{A^{\prime} C^{\prime}}{A C}-\frac{B^{\prime} C^{\prime}}{B C}\right)+\frac{1}{C}\left(\frac{C^{\prime \prime}}{B^{2}}-\frac{\ddot{C}}{A^{2}}\right)=0
$$

Thorne [27] defined C-energy for the cylindrical symmetric spacetime

$$
m(t, r)=\tilde{E}(t, r)=\frac{1}{8}\left(1-l^{-2} \nabla^{\alpha} \tilde{r} \nabla_{\alpha} \tilde{r}\right)
$$

The circumference radius $\rho$, specific length $l$ and areal radius $\tilde{r}$ can be defined as

$$
\rho^{2}=\xi_{(1) a} \xi_{(1)}^{a}, \quad l^{2}=\xi_{(2) a} \xi_{(2)}^{a}, \quad \tilde{r}=\rho l
$$

where $\xi_{(1)}=\frac{\partial}{\partial \theta}, \xi_{(2)}=\frac{\partial}{\partial z}$ are the Killing vectors and $\tilde{E}(t, r)$ represents the gravitational energy per unit specific length of the cylinder. The specific energy of the cylinder [28] in the interior region with electromagnetic field can be written as follows

$$
m(t, r)=E^{\prime}(t, r)=\frac{1}{8}\left[1+\left(\frac{\dot{C}}{A}\right)^{2}-\left(\frac{C^{\prime}}{B}\right)^{2}\right]+\frac{s^{2}}{2 C}
$$


The dynamical equations can be obtained from the contracted Bianchi identities $\left(T^{-\alpha \beta}+E^{-\alpha \beta}\right)_{; \beta}=0$ yielding the following two equations

$$
\begin{aligned}
\dot{\mu}+q^{\prime} A+\left(2 \frac{A^{\prime}}{A}+\frac{B^{\prime}}{B}+\frac{C^{\prime}}{C}\right) q A+(\mu+p)\left(\frac{\dot{B}}{B}+\frac{\dot{C}}{C}\right) & =0, \\
p^{\prime}+\dot{q} \frac{A}{B^{2}}+\left(3 \frac{\dot{B}}{B}+\frac{\dot{C}}{C}\right) q \frac{B^{2}}{A}+(\mu+p) \frac{A^{\prime}}{A}-\left(C E^{\prime}+E C^{\prime}\right) \frac{E}{C} & =0 .
\end{aligned}
$$

\section{Junction Conditions}

In this section, we take a timelike $3 D$ hypersurface $\Sigma$ which splits two $4 D$ manifolds $V^{-}$and $V^{+}$as interior and exterior regions respectively. The interior region is defined in Eq.(1) while the exterior region is described by cylindrically symmetric manifold [29] in the retarded time coordinate as

$$
d s_{+}^{2}=-\left(-\frac{2 M(\nu)}{R}+\frac{Q^{2}(\nu)}{R^{2}}\right) d \nu^{2}-2 d \nu d R+R^{2}\left(d \theta^{2}+\gamma^{2} d z^{2}\right),
$$

where $\gamma^{2}=-\frac{\Lambda}{3}, \Lambda$ being the cosmological constant and $M(\nu)$ is the total mass, $Q(\nu)$ is the charge inside the boundary surface $\Sigma$ and $\nu$ is the retarded time. For smooth matching of the interior and exterior regions, Darmois conditions [24] can be stated as follows:

1. The continuity of the line elements over $\Sigma$

$$
\left(d s_{-}^{2}\right)_{\Sigma}=\left(d s_{+}^{2}\right)_{\Sigma}=\left(d s^{2}\right)_{\Sigma} .
$$

2. The continuity of the extrinsic curvature over $\Sigma$

$$
\left[K_{i j}\right]=K_{i j}^{+}-K_{i j}^{-}=0, \quad(i, j=0,2,3) .
$$

The boundary surface $\Sigma$ in terms of interior and exterior coordinates can be defined as

$$
\begin{aligned}
f_{-}(t, r) & =r-r_{\Sigma}=0, \\
f_{+}(\nu, R) & =R-R\left(\nu_{\Sigma}\right)=0,
\end{aligned}
$$

where $r_{\Sigma}$ is a constant. Using Eqs.(26) and (27), the interior and exterior metrics take the following form over $\Sigma$

$$
\left(d s_{-}^{2}\right)_{\Sigma}=-A^{2}\left(t, r_{\Sigma}\right) d t^{2}+C^{2}\left(t, r_{\Sigma}\right) d \theta^{2}+d z^{2}
$$




$$
\left(d s_{+}^{2}\right)_{\Sigma}=-\left(-\frac{2 M(\nu)}{R(\nu)_{\Sigma}}+\frac{Q^{2}(\nu)}{R(\nu)_{\Sigma}^{2}}+2 \frac{d R(\nu)_{\Sigma}}{d \nu}\right) d \nu^{2}+R_{\Sigma}^{2}\left(d \theta^{2}+\gamma^{2} d z^{2}\right)
$$

The first condition implies

$$
\frac{d t}{d \tau}=A\left(t, r_{\Sigma}\right)^{-1}, \quad C\left(t, r_{\Sigma}\right)=R_{\Sigma}(\nu)=\frac{1}{\gamma}
$$

yielding the radius of $\Sigma$ in both interior and exterior coordinates

$$
\left(\frac{d \nu}{d \tau}\right)^{-2}=\left(-\frac{2 M(\nu)}{R_{\Sigma}}+\frac{Q(\nu)^{2}}{R_{\Sigma}^{2}}+2 \frac{d R_{\Sigma}}{d \nu}\right)
$$

The outward unit normals to $\Sigma$ are found by using Eqs.(26) and (27)

$$
\begin{aligned}
& n_{\alpha}^{-}=\left(0, B\left(t, r_{\Sigma}\right), 0,0\right) \\
& n_{\alpha}^{+}=\left(-\frac{2 M(\nu)}{R_{\Sigma}}+\frac{Q(\nu)^{2}}{R_{\Sigma}^{2}}+2 \frac{d R_{\Sigma}}{d \nu}\right)^{-\frac{1}{2}}\left(-\frac{d R_{\Sigma}}{d \nu}, 1,0,0\right) .
\end{aligned}
$$

The non-vanishing components of extrinsic curvature in terms of interior and exterior coordinates are

$$
\begin{aligned}
& K_{00}^{-}=-\left[\frac{A^{\prime}}{A B}\right]_{\Sigma}, \quad K_{22}^{-}=\left[\frac{C C^{\prime}}{B}\right]_{\Sigma}, \\
& K_{00}^{+}=\left[\left(\frac{d^{2} \nu}{d \tau^{2}}\right)\left(\frac{d \nu}{d \tau}\right)^{-1}-\left(\frac{d \nu}{d \tau}\right)\left(\frac{M}{R^{2}}-\frac{Q^{2}}{R^{3}}\right)\right]_{\Sigma}, \\
& K_{22}^{+}=\left[R\left(\frac{d R}{d \tau}\right)-\left(\frac{d \nu}{d \tau}\right)\left(2 M-\frac{Q^{2}}{R}\right)\right]_{\Sigma}=\gamma^{2} K_{33}^{+} .
\end{aligned}
$$

Using the continuity of extrinsic curvature and Eqs.(30) and (31), we get

$$
\begin{array}{r}
M(\nu)=\frac{R}{2}\left[\left(\frac{\dot{R}}{A}\right)^{2}-\left(\frac{R^{\prime}}{B}\right)^{2}\right]+\frac{Q^{2}}{2 R} . \\
E^{\prime}-M \stackrel{\Sigma}{=} \frac{1}{8}, \quad p \stackrel{\Sigma}{=}(q B) .
\end{array}
$$

where $s \stackrel{\Sigma}{=} Q$ has been used. This shows that the difference of both masses at the boundary $\Sigma$ turns out to be $\frac{1}{8}$ which is zero in spherical case. This is the least satisfactory condition of the C-energy where the isotropic pressure is equal to the radial heat-flow. It is mentioned here that pressure at the boundary will be zero only if there is no dissipation, i.e., $q \stackrel{\Sigma}{=} 0$. 


\section{The Perturbation Scheme}

A stable stationary black hole solution under perturbation indicates the final state of the dynamical evolution of a gravitating system. This scheme is also used to identify basic properties of black hole solution [30. Here we perturb the field equations, Bianchi identities and all the material functions upto first order in $\lambda$ such that the fluid is in hydrostatic equilibrium form. These are given by [5]-[7]

$$
\begin{aligned}
A(t, r) & =A_{0}(r)+\lambda T(t) a(r), \\
B(t, r) & =B_{0}(r)+\lambda T(t) b(r), \\
C(t, r) & =r B(t, r)[1+\lambda T(t) \bar{c}(r)], \\
E(t, r) & =E_{0}(r)+\lambda T(t) e(r), \\
\mu(t, r) & =\mu_{0}(r)+\lambda \bar{\mu}(t, r), \\
p(t, r) & =p_{0}(r)+\lambda \bar{p}(t, r), \\
q(t, r) & =\lambda \bar{q}(t, r), \\
m(t, r) & =m_{0}(r)+\lambda \bar{m}(t, r),
\end{aligned}
$$

where $0<\lambda \ll 1$. The static configuration of Eqs.(12)-(14) and (17) is obtained by using Eqs.(39)-(45) as follows

$$
\begin{aligned}
4 \pi\left(2 \mu_{0}+\pi E_{0}^{2}\right) & =\frac{1}{B_{0}^{2}}\left[\left(\frac{B_{0}^{\prime}}{B_{0}}\right)^{2}-\frac{1}{r} \frac{B_{0}^{\prime}}{B_{0}}-\frac{B_{0}^{\prime \prime}}{B_{0}}\right] \\
4 \pi\left(2 p_{0}-\pi E_{0}^{2}\right) & =\frac{1}{B_{0}^{2}} \frac{A_{0}^{\prime}}{A_{0}}\left(\frac{B_{0}^{\prime}}{B_{0}}+\frac{1}{r}\right), \\
0 & =\frac{1}{B_{0}^{2}}\left[\left(\frac{A_{0}^{\prime}}{A_{0}}-\frac{B_{0}^{\prime}}{B_{0}}\right)\left(\frac{1}{r}+\frac{B_{0}^{\prime}}{B_{0}}\right)+\left(\frac{1}{r} \frac{B_{0}^{\prime}}{B_{0}}+\frac{B_{0}^{\prime \prime}}{B_{0}}\right)\right]
\end{aligned}
$$

The corresponding perturbed field equations become

$$
\begin{aligned}
8 \pi\left(\bar{\mu}+\pi E_{0} T e\right) & =-\frac{T}{B_{0}^{2}}\left[\left(\frac{b}{B_{0}}-\bar{c}\right)\left(\frac{B_{0}^{\prime}}{B_{0}}\right)^{2}+\frac{1}{B_{0}}\left(\left(\bar{c} B_{0}^{\prime}\right)^{\prime}+\frac{\left(r b^{\prime}\right)^{\prime}}{r}\right)\right. \\
& \left.+\frac{2}{r}\left(\bar{c}^{\prime}+\frac{r \bar{c}^{\prime \prime}}{2}\right)-\frac{B_{0}^{\prime}}{B_{0}}\left(\frac{2 b^{\prime}}{B_{0}}-\frac{\bar{c}}{r}\right)\right]-\frac{4 \pi T}{B_{0}^{2}}\left(\frac{3 b}{B_{0}}+\bar{c}\right) \\
& \times\left(2 \mu_{0}+\pi E_{0}^{2}\right), \\
8 \pi B_{0}^{2} \bar{q} & =\left[\left(\frac{b}{A_{0} B_{0}}\right)^{\prime}+\left(\frac{\bar{c}}{A_{0}}\right)^{\prime}+\left(\frac{1}{r}+\frac{B_{0}^{\prime}}{B_{0}}\right)\left(\frac{\bar{c}}{A_{0}}\right)\right] \dot{T},
\end{aligned}
$$




$$
\begin{aligned}
8 \pi\left(\bar{p}-\pi E_{0} T e\right) & =-\frac{\ddot{T}}{A_{0}^{2}}\left(\frac{b}{B_{0}}+\bar{c}\right)+\frac{T}{B_{0}^{2}}\left[\left(\frac{1}{r}+\frac{B_{0}^{\prime}}{B_{0}}\right)\left(\frac{a}{A_{0}}\right)^{\prime}\right. \\
& \left.+\frac{A_{0}^{\prime}}{A_{0}}\left(\frac{b}{B_{0}}+\bar{c}\right)^{\prime}\right]-8 \pi \frac{T b}{B_{0}}\left(2 p_{0}-\pi E_{0}^{2}\right) \\
0 & =-\frac{1}{A_{0}^{2} B_{0}}\left(b+\bar{c} B_{0}\right) \ddot{T}+\frac{T}{B_{0}^{2}}\left[\frac { b } { r B _ { 0 } } \left(\frac{1}{A_{0}}-\frac{1}{B_{0}}+r\left(\frac{B_{0}^{\prime}}{B_{0}}\right)^{2}\right.\right. \\
& \left.+\frac{B_{0}^{\prime}}{B_{0}}\right)+\frac{b^{\prime}}{B_{0}}\left(\frac{1}{A_{0}}-\frac{1}{B_{0}}+\frac{2}{r}-\frac{B_{0}^{\prime}}{A_{0}}-\frac{B_{0}}{r A_{0}}\right)+\left(2 \bar{c}^{\prime}\right. \\
& \left.-\frac{a A_{0}^{\prime}}{A_{0}^{2}}\right)\left(\frac{1}{r}+\frac{B_{0}^{\prime}}{B_{0}}\right)+\frac{a^{\prime}}{A_{0}}\left(\frac{1}{r}+\frac{B_{0}^{\prime}}{B_{0}}\right)+\frac{b^{\prime \prime}}{B_{0}}+\frac{\bar{c}}{r}\left(\frac{1}{A_{0}}\right. \\
& \left.\left.-\frac{1}{B_{0}}+2 \frac{B_{0}^{\prime}}{B_{0}}+\frac{r B_{0}^{\prime \prime}}{B_{0}}\right)\right] .
\end{aligned}
$$

Bianchi identities (21) and (22) for static and perturbed configuration yields

$$
\begin{aligned}
& p_{0}^{\prime}+\left(\mu_{0}+p_{0}\right) \frac{A_{0}^{\prime}}{A_{0}}-E_{0}\left[E_{0}\left(\frac{1}{r}+\frac{B_{0}^{\prime}}{B_{0}}\right)+E_{0}^{\prime}\right]=0 \\
& \overline{\dot{\mu}}+\bar{q}^{\prime} A_{0}+\left(2 \frac{A_{0}^{\prime}}{A_{0}}+\frac{B_{0}^{\prime}}{B_{0}}+\frac{1}{r}\right) \bar{q} A_{0}+\left(\frac{2 b}{B_{0}}+\bar{c}\right)\left(\mu_{0}+p_{0}\right) \dot{T}=0,(55) \\
& \bar{p}^{\prime}+\dot{\bar{q}} \frac{B_{0}^{2}}{A_{0}}+(\bar{p}+\bar{\mu})\left(\frac{A_{0}^{\prime}}{A_{0}}\right)+\left(p_{0}+\mu_{0}\right)\left(\frac{a}{A_{0}}\right)^{\prime} T-E_{0}\left[\left(E_{0} \bar{c}\right)^{\prime}\right. \\
+ & \left.\left(E_{0} \bar{c}+e\right)\left(\frac{1}{r}+\frac{B_{0}^{\prime}}{B_{0}}\right)+\frac{1}{r B_{0}}\left(r b E_{0}\right)^{\prime}+e^{\prime}\right]=0
\end{aligned}
$$

Using Eq.(51) in (55) and then integrating, it follows

$$
\bar{\mu}=-\left(\frac{2 b}{B_{0}}+\bar{c}\right)\left(\mu_{0}+p_{0}\right) T-\frac{1}{8 \pi} \frac{A_{0}}{B_{0}^{2}} \phi(r) T,
$$

where

$$
\begin{aligned}
\phi(r) & =\left\{\left(\frac{1}{r}+\frac{2 A_{0}^{\prime}}{A_{0}}\right)\left[\left(\frac{b}{A_{0} B_{0}}\right)^{\prime}+\left(\frac{\bar{c}}{A_{0}}\right)^{\prime}+\left(\frac{1}{r}+\frac{B_{0}^{\prime}}{B_{0}}\right)\left(\frac{\bar{c}}{A_{0}}\right)\right]\right. \\
& \left.+\left[\left(\frac{b}{A_{0} B_{0}}\right)^{\prime}+\left(\frac{\bar{c}}{A_{0}}\right)^{\prime}+\left(\frac{1}{r}+\frac{B_{0}^{\prime}}{B_{0}}\right)\left(\frac{\bar{c}}{A_{0}}\right)\right]^{\prime}\right\} .
\end{aligned}
$$


Similarly, the static configuration of Eq.(20) turn out to be

$$
m_{0}=\frac{1}{8}\left[1-\left(\frac{1}{r}+\frac{B_{0}^{\prime}}{B_{0}}\right)^{2}+16 \pi^{2} E_{0}^{2} B_{0} r\right] .
$$

The above equation gives the total energy entrapped inside $\Sigma$. From Eqs.(54) and (59), we have

$$
\begin{aligned}
& \frac{A_{0}^{\prime}}{A_{0}}=-\frac{1}{\mu_{0}+p_{0}}\left[p_{0}^{\prime}-E_{0}\left[E_{0}\left(\frac{1}{r}+\frac{B_{0}^{\prime}}{B_{0}}\right)+E_{0}^{\prime}\right]\right], \\
& \frac{B_{0}^{\prime}}{B_{0}}=-\frac{1}{r}+\sqrt{1-8 m_{0}+16 \pi^{2} E_{0}^{2} B_{0} r} .
\end{aligned}
$$

The second order linear differential equation can be obtained from Eqs.(51) and (52) with the help of junction condition and the relation $p_{0} \stackrel{\Sigma}{=} 0$

$$
\ddot{T}+2 \beta \dot{T}-\alpha T \stackrel{\Sigma}{=} 0,
$$

where

$$
\begin{aligned}
\alpha(r) & \stackrel{\Sigma}{=}\left(\frac{A_{0}}{B_{0}}\right)^{2}\left(\frac{b}{B_{0}}+\bar{c}\right)^{-1}\left[\left(\frac{1}{r}+\frac{B_{0}^{\prime}}{B_{0}}\right)\left(\frac{a}{A_{0}}\right)^{\prime}\right. \\
& \left.+\frac{A_{0}^{\prime}}{A_{0}}\left(\frac{b}{B_{0}}+\bar{c}\right)^{\prime}+8 \pi^{2} E_{0} B_{0}\left(e b_{0}+b E_{0}\right)\right] \\
\beta(r) & \stackrel{\Sigma}{=} \frac{1}{2} \frac{A_{0}^{2}}{B_{0}}\left(\frac{b}{B_{0}}+\bar{c}\right)^{-1}\left[\left(\frac{b}{A_{0} B_{0}}\right)^{\prime}+\left(\frac{\bar{c}}{A_{0}}\right)^{\prime}+\left(\frac{1}{r}+\frac{B_{0}^{\prime}}{B_{0}}\right)\left(\frac{\bar{c}}{A_{0}}\right)\right] 6
\end{aligned}
$$

The general solution of Eq.(62) is given by

$$
T(t)=-\exp \left[\left(-\beta_{\Sigma}+\sqrt{\alpha_{\Sigma}+\beta_{\Sigma}^{2}}\right) t\right],
$$

where for the sake of real solution and range of instability, we assume that $\alpha_{\Sigma}>0$ and $\beta_{\Sigma}<0$. We see that the system starts collapsing at $t=-\infty$ with $T(-\infty)=0$ in static position and it continues to collapse with the increase of $t$. 


\section{Dynamical Instability}

Here we discuss the dynamical instability at Newtonian and post-Newtonian regimes. We relate $\Gamma$ in terms of $\bar{p}$ and $\bar{\mu}$ as the ratio of specific heats

$$
\bar{p}=\Gamma \frac{p_{0}}{\mu_{0}+p_{0}} \bar{\mu}
$$

$\Gamma$ is taken to be constant throughout the dynamical instability. Using Eq.(57) in the above equation, we obtain

$$
\bar{p}=-p_{0} \Gamma\left(\frac{2 b}{B_{0}}+\bar{c}\right) T-\frac{1}{8 \pi} \frac{A_{0}}{B_{0}^{2}} \Gamma \frac{p_{0}}{\mu_{0}+p_{0}} \phi(r) T .
$$

Also, from Eq.(152), it follows that

$$
\begin{aligned}
\left(\frac{a}{A_{0}}\right)^{\prime} & =\left(\frac{1}{r}+\frac{B_{0}^{\prime}}{B_{0}}\right)^{-1}\left[-8 \pi\left(\frac{2 b}{B_{0}}+\bar{c}\right) \Gamma p_{0} B_{0}^{2}-\Gamma \frac{p_{0}}{\mu_{0}+p_{0}} A_{0} \phi(r) T\right. \\
& +\frac{B_{0}^{2}}{A_{0}}\left(\frac{b}{B_{0}}+\bar{c}\right) \frac{\ddot{T}}{T}-\frac{A_{0}^{\prime}}{A_{0}}\left(\frac{b}{B_{0}}+\bar{c}\right)^{\prime}-8 \pi^{2} E_{0} B_{0}\left(e B_{0}+b E_{0}\right) \\
& \left.+16 \pi b B_{0} p_{0}\right] .
\end{aligned}
$$

Making use of Eqs.(51), (57), (67) and (68) in (56), we get the collapse equation

$$
\begin{aligned}
& {\left[-p_{0} \Gamma\left(\frac{2 b}{B_{0}}+\bar{c}\right)-\frac{1}{8 \pi} \frac{A_{0}}{B_{0}^{2}} \Gamma \frac{p_{0}}{\mu_{0}+p_{0}} \phi(r)\right]^{\prime}-\left[( \frac { 2 b } { B _ { 0 } } + \overline { c } ) \left(\mu_{0}+p_{0}\right.\right.} \\
+ & \left.\left.\Gamma p_{0}\right)-\frac{1}{8 \pi} \frac{A_{0}}{B_{0}^{2}}\left(\Gamma \frac{p_{0}}{\mu_{0}+p_{0}}+1\right) \phi(r)\right] \frac{A_{0}^{\prime}}{A_{0}}+\left(\mu_{0}+p_{0}\right)\left(\frac{1}{r}+\frac{B_{0}^{\prime}}{B_{0}}\right)^{-1} \\
\times & {\left[-8 \pi\left(\frac{2 b}{B_{0}}+\bar{c}\right) \Gamma p_{0} B_{0}^{2}-\Gamma \frac{p_{0}}{\mu_{0}+p_{0}} A_{0} \phi(r)+\frac{B_{0}^{2}}{A_{0}}\left(\frac{b}{B_{0}}+\bar{c}\right) \frac{\ddot{T}}{T}\right.} \\
- & \left.\frac{A_{0}^{\prime}}{A_{0}}\left(\frac{b}{B_{0}}+\bar{c}\right)^{\prime}-8 \pi^{2} E_{0} B_{0}\left(e B_{0}+b E_{0}\right)+16 \pi b B_{0} p_{0}\right]-E_{0}\left[e^{\prime}\right. \\
+ & \left.\left(E_{0} \bar{c}\right)^{\prime}+\left(E_{0} \bar{c}+e\right)\left(\frac{1}{r}+\frac{B_{0}^{\prime}}{B_{0}}\right)+\frac{1}{r B_{0}}\left(r b E_{0}\right)^{\prime}\right]+\frac{1}{4 \pi} \frac{B_{0}}{A_{0}^{3}} \beta \frac{\ddot{T}}{T}=0 .
\end{aligned}
$$

Now we discuss the dynamical instability at Newtonian and $\mathrm{pN}$ regimes in the following cases of vanishing and non-vanishing heat flow. 


\subsection{Non-Dissipative Perturbation}

In this case, we assume that the fluid is non-dissipative, i.e., $q=0$. As a result, Eq. (51) can be written as

$$
\left(\frac{b}{A_{0} B_{0}}\right)^{\prime}+\left(\frac{\bar{c}}{A_{0}}\right)^{\prime}+\left(\frac{1}{r}+\frac{B_{0}^{\prime}}{B_{0}}\right)\left(\frac{\bar{c}}{A_{0}}\right)=0 .
$$

This is trivially satisfied if both the terms are taken to be identically zero, i.e.,

$$
\left(\frac{b}{A_{0} B_{0}}\right)^{\prime}=0, \quad\left(\frac{\bar{c}}{A_{0}}\right)^{\prime}+\left(\frac{1}{r}+\frac{B_{0}^{\prime}}{B_{0}}\right)\left(\frac{\bar{c}}{A_{0}}\right)=0 .
$$

Integration of the first term gives

$$
b=A_{0} B_{0}
$$

while the remaining two terms yield

$\bar{c}^{\prime}=-\left(\frac{p_{0}^{\prime}}{\mu_{0}+p_{0}}-\frac{E_{0}^{2}+E_{0} E_{0}^{\prime}+8 \pi^{2} r E_{0}^{4}}{\mu_{0}+p_{0}}\right) \bar{c}-\bar{c} \sqrt{1-8 m_{0}+16 \pi^{2} E_{0}^{2} B_{0} r}$.

Since there is no dissipation, thus Eqs.(58) and (64) gives $\phi(r)=0$ and $\beta(r)=0$ respectively. Now using these results and Eqs.(72) and (73) in the collapse equation (69), we obtain

$$
\begin{array}{ll} 
& -\left(2 A_{0}+\bar{c}\right) \Gamma p_{0}^{\prime}-\Gamma p_{0}\left[\left(2 A_{0}+\bar{c}\right)\left(-\frac{p_{0}^{\prime}}{\mu_{0}+p_{0}}+\frac{E_{0}^{2}+E_{0} E_{0}^{\prime}+8 \pi^{2} r E_{0}^{4}}{\mu_{0}+p_{0}}\right)\right. \\
- & \left.\bar{c} \sqrt{1-8 m_{0}+16 \pi^{2} E_{0}^{2} B_{0} r}\right]-\left(2 A_{0}+\bar{c}\right)\left(\mu_{0}+p_{0}+\Gamma p_{0}\right)\left(-\frac{p_{0}^{\prime}}{\mu_{0}+p_{0}}\right. \\
+ & \left.\frac{E_{0}^{2}+E_{0} E_{0}^{\prime}+8 \pi^{2} r E_{0}^{4}}{\mu_{0}+p_{0}}\right)+\left(\mu_{0}+p_{0}\right)\left(\sqrt{1-8 m_{0}+16 \pi^{2} E_{0}^{2} B_{0} r}\right)^{-1} \\
\times & \left\{-8 \pi\left(2 A_{0}+\bar{c}\right) \Gamma p_{0} B_{0}^{2}-8 \pi^{2} E_{0}\left(e+A_{0} E_{0}\right) B_{0}^{2}+16 \pi p_{0} B_{0}^{2}+\frac{B_{0}^{2}}{A_{0}}\left(A_{0}+\bar{c}\right)\right. \\
\times & \alpha_{\Sigma}-A_{0}\left(-\frac{p_{0}^{\prime}}{\mu_{0}+p_{0}}+\frac{E_{0}^{2}+E_{0} E_{0}^{\prime}+8 \pi^{2} r E_{0}^{4}}{\mu_{0}+p_{0}}-\left(-\frac{p_{0}^{\prime}}{\mu_{0}+p_{0}}\right.\right. \\
+ & \left.\frac{E_{0}^{2}+E_{0} E_{0}^{\prime}+8 \pi^{2} r E_{0}^{4}}{\mu_{0}+p_{0}}\right)\left[-\frac{p_{0}^{\prime}}{\mu_{0}+p_{0}}+\frac{E_{0}^{2}+E_{0} E_{0}^{\prime}+8 \pi^{2} r E_{0}^{4}}{\mu_{0}+p_{0}} \bar{c}\right. \\
- & \left.\left.\bar{c} \sqrt{1-8 m_{0}+16 \pi^{2} E_{0}^{2} B_{0} r}\right]\right\}-E_{0}\left[e^{\prime}+E_{0}^{\prime} \bar{c}+A_{0} E_{0}^{\prime}+\frac{A_{0} E_{0}}{r}+\left(E_{0} \bar{c}\right.\right.
\end{array}
$$




$$
\begin{aligned}
& + \text { e) }\left(\sqrt{1-8 m_{0}+16 \pi^{2} E_{0}^{2} B_{0} r}\right)-\bar{c} E_{0} \sqrt{1-8 m_{0}+16 \pi^{2} E_{0}^{2} B_{0} r} \\
& +E_{0} \bar{c}\left(-\frac{p_{0}^{\prime}}{\mu_{0}+p_{0}}+\frac{E_{0}^{2}+E_{0} E_{0}^{\prime}+8 \pi^{2} r E_{0}^{4}}{\mu_{0}+p_{0}}\right)+E_{0} A_{0}\left(-\frac{p_{0}^{\prime}}{\mu_{0}+p_{0}}\right. \\
& \left.\left.+\frac{E_{0}^{2}+E_{0} E_{0}^{\prime}+8 \pi^{2} r E_{0}^{4}}{\mu_{0}+p_{0}}-\frac{1}{r}+\sqrt{1-8 m_{0}+16 \pi^{2} E_{0}^{2} B_{0} r}\right)\right]=0 .
\end{aligned}
$$

\section{Newtonian limit}

For the instability condition at Newtonian limit, we take $A_{0}=1, B_{0}=1$, $\mu_{0} \gg p_{0}$ and neglecting the relativistic effects. Equation (74) yields

$$
-2 p_{0}^{\prime} \Gamma+2 p_{0}^{\prime}-3\left(E_{0}^{2}+8 \pi^{2} r E_{0}^{4}+E_{0} E_{0}^{\prime}\right)+\left(1-8 \pi^{2} r E_{0}^{2}\right) \alpha_{\Sigma} \mu_{0}=0,
$$

Thus the instability condition for the isotropic fluid in the cylindrically symmetric spacetime is given by

$$
\Gamma<1+\left[\frac{3\left(E_{0}^{2}+8 \pi^{2} r E_{0}^{4}+E_{0} E_{0}^{\prime}\right)}{2\left|p_{0}^{\prime}\right|}\right],
$$

where we assume $p_{0}^{\prime}<0$. This equation shows that the electromagnetic field increases the instability of the collapsing fluid.

\section{Post-Newtonian limit}

In this limit, we use $A_{0}=1-\frac{m_{0}}{r}, B_{0}=1-\frac{m_{0}}{r}$ and the relativistic effects upto order $\frac{m_{0}}{r}$. Thus we have

$$
\begin{aligned}
& -2 p_{0}^{\prime} \Gamma+2 p_{0}^{\prime}-3\left(E_{0}^{2}+8 \pi^{2} r E_{0}^{4}+E_{0} E_{0}^{\prime}\right)+\left(1-8 \pi^{2} r E_{0}^{2}\right)\left[16 \pi p_{0} \mu_{0}\right. \\
\times & \left.(1-\Gamma)-8 \pi^{2} \mu_{0} E_{0}\left(e+E_{0}\right)+(1+\bar{c}) \alpha_{\Sigma} \mu_{0}\right]-E_{0}\left[e^{\prime}+e\left(1+8 \pi^{2} r E_{0}^{2}\right)\right. \\
+ & \left.E_{0}^{\prime} \bar{c}+(1+\bar{c})\left(E_{0}^{2}+8 \pi^{2} r E_{0}^{4}+E_{0} E_{0}^{\prime}\right) \frac{E_{0}}{\mu_{0}}\right]=0 .
\end{aligned}
$$

Since the system starts collapsing as $t \rightarrow-\infty$, i.e., $\dot{T}<0$, it follows from Eq.(51) that $\bar{c}>0$. Hence the instability condition at $\mathrm{pN}$ limit becomes

$$
\begin{gathered}
\Gamma<1+\frac{1}{2\left|p_{0}^{\prime}\right|}\left[3\left(E_{0}^{2}+8 \pi^{2} r E_{0}^{4}+E_{0} E_{0}^{\prime}\right)+8 \pi^{2} \mu_{0} e E_{0}^{2}+E_{0}\left(e^{\prime}\right.\right. \\
\left.\left.+\quad e\left(1+8 \pi^{2} r E_{0}^{2}\right)+E_{0}^{\prime} \bar{c}\right)\right]-\frac{1}{2\left|p_{0}^{\prime}\right|}\left(16 \pi p_{0} \mu_{0}+(1+\bar{c}) \alpha_{\Sigma} \mu_{0}\right) .
\end{gathered}
$$


This shows that the instability range is increased by the first three terms in the square brackets and is decreased by the last term. It is mentioned here that the first term in the square brackets is the Newtonian term while the remaining terms are the relativistic corrections.

\subsection{Dissipative Perturbation}

When $q \neq 0$, the perturbed solution corresponding to Eq.(72) becomes

$$
b(r)=A_{0} B_{0}[1+\eta f(r)],
$$

where $\eta>0$ is of the order $\frac{m_{0}}{r}$. Using the above solution in Eqs.(58) and (64), $\phi(r)$ and $\beta(r)$ turn out to be of order $\frac{m_{0}}{r}$.

\section{Newtonian limit}

Applying the same approach as defined in the non-dissipative case and using the values $A_{0}=1, B_{0}=1, \mu_{0} \gg p_{0}$, Eq.(69) at Newtonian limit reduces to

$$
\begin{aligned}
& -2(1+\eta f) p_{0}^{\prime} \Gamma+2(1+\eta f) p_{0}^{\prime}-3(1+\eta f)\left(E_{0}^{2}+E_{0} E_{0}^{\prime}+8 \pi^{2} r E_{0}^{4}\right) \\
+ & \left(1-8 \pi^{2} r E_{0}^{2}\right)(1+\eta f) \mu_{0} \alpha_{\Sigma}+\frac{\eta f^{\prime}}{4 \pi} \alpha_{\Sigma}=0
\end{aligned}
$$

In general, the heat flow $q>0$ and $\dot{T}<0$ for a collapsing fluid, implying $\bar{c}<0$ and $f^{\prime}<0$ from Eq.(51). Consequently the instability condition becomes

$$
\Gamma<1+\left[\frac{3\left(E_{0}^{2}+E_{0} E_{0}^{\prime}+8 \pi^{2} r E_{0}^{4}\right)}{2\left|p_{0}^{\prime}\right|}+\frac{\eta\left|f^{\prime}\right| \alpha_{\Sigma}}{8 \pi\left|p_{0}^{\prime}\right|(1+\eta|f|)}\right] .
$$

We note that the electromagnetic field as well as dissipation enhance the instability range, which supports the result for spherical case [5].

\section{Post-Newtonian limit}

Here we take $A_{0}=1-\frac{m_{0}}{r}, \quad B_{0}=1-\frac{m_{0}}{r}$ and consider the relativistic correction terms upto order $\frac{m_{0}}{r}$. The collapse equation at $\mathrm{pN}$ limit yields

$$
\begin{array}{ll} 
& -(2+2 \eta f+\bar{c}) p_{0}^{\prime} \Gamma+(2+2 \eta f+\bar{c}) p_{0}^{\prime}-(3+3 \eta f+\bar{c})\left(E_{0}^{2}+E_{0} E_{0}^{\prime}\right. \\
+ & \left.8 \pi^{2} r E_{0}^{4}\right)+\frac{1}{4 \pi} \eta f^{\prime} \alpha_{\Sigma}-\left(1-8 \pi^{2} r E_{0}^{2}\right)\left[8 \pi^{2} \mu_{0} E_{0} e+8 \pi(2+2 \eta f+\bar{c})\right. \\
\times & p_{0} \mu_{0} \Gamma-(1+\eta f+\bar{c}) \alpha_{\Sigma} \mu_{0}-8 \pi(1+\eta f)\left(2 p_{0}-\pi E_{0}\right) \mu_{0}+\left(\eta f^{\prime}+\bar{c}^{\prime}\right) \\
\times & \left.\left(-p_{0}^{\prime}+E_{0}^{2}+E_{0} E_{0}^{\prime}+8 \pi^{2} r E_{0}^{4}\right)\right]-E_{0}\left[e^{\prime}+\left(1+8 \pi^{2} r E_{0}^{2}\right)\left(e+E_{0} \bar{c}\right)\right.
\end{array}
$$




$$
\left.+E_{0} \bar{c}^{\prime}+E_{0}^{\prime} \bar{c}+E_{0}\left(\eta f^{\prime}+\frac{(1+\eta f)}{\mu_{0}}\left(E_{0}^{2}+E_{0} E_{0}^{\prime}+8 \pi^{2} r E_{0}^{4}\right)\right)\right]=0 .
$$

Using the same arguments as given in Newtonian limit, we have the instability condition

$$
\begin{aligned}
& \Gamma<1+\left\{\frac{3\left(E_{0}^{2}+E_{0} E_{0}^{\prime}+8 \pi^{2} r E_{0}^{4}\right)}{2\left|p_{0}^{\prime}\right|}+\frac{1}{(1+\eta|f|)} \frac{\eta\left|f^{\prime}\right|}{8 \pi\left|p_{0}^{\prime}\right|} \alpha_{\Sigma}\right. \\
+ & \frac{4 \pi^{2} \mu_{0} E_{0} e}{(1+\eta|f|)\left|p_{0}^{\prime}\right|}+\frac{E_{0}}{(1+\eta|f|)\left|p_{0}^{\prime}\right|}\left[e^{\prime}+\bar{c} \alpha_{\Sigma} \mu_{0}+\eta\left|f^{\prime}\right| E_{0}^{\prime}\right. \\
+ & \left.\left.e\left(1+8 \pi^{2} r E_{0}^{2}\right)\right]\right\}-E_{0} \frac{\left(E_{0} \bar{c}\right)^{\prime}+E_{0} \bar{c}}{(1+\eta|f|)\left|p_{0}^{\prime}\right|}-\frac{\alpha_{\Sigma} \mu_{0}}{\left|p_{0}^{\prime}\right|} \\
- & \frac{4 \pi\left(2 p_{0}+\pi E_{0}\right) \mu_{0}}{\left|p_{0}^{\prime}\right|}-\eta\left|f^{\prime}\right|\left(1+\frac{E_{0}^{2}+E_{0} E_{0}^{\prime}+8 \pi^{2} r E_{0}^{4}}{\left|p_{0}^{\prime}\right|}\right) .
\end{aligned}
$$

This implies that the range of instability is increased by Newtonian term and relativistic terms in the square brackets while it is diminished by the last four terms.

\section{Summary}

This paper has been addressed to discuss the dynamical instability of cylindrically symmetric non-viscous heat conducting isotropic fluid with electromagnetic field. For spherically symmetric spacetime [1, it has been shown that when a star is compressed or expanded adiabatically, its dynamical instability depends on the numerical value of the adiabatic index, i.e., $\frac{4}{3}$. If $\Gamma>\frac{4}{3}$, the pressure in the star is strong enough than the weight of the layers which makes the star stable. On the other hand, If $\Gamma<\frac{4}{3}$, the weight increases very fast than the pressure and the star collapses resulting a dynamical instability.

We have found that for the cylindrically symmetric case, the dynamical instability depends on the critical value 1 . When $\Gamma>1$, the stability of the system is obtained while $\Gamma<1$ yields a dynamical instability. Further, we have studied the role played by the electromagnetic field and dissipation in the early stages of dynamical instability. It is interesting to mention here that different ranges of instability may lead to different patterns of evolution of stars. We note from Eqs.(76) and (81) that electromagnetic field as well as dissipation make the system more unstable at Newtonian regime. This 
supports the fact that different physical aspects of the fluid has a great relevance in the evolution as well as structure formation of self-gravitating objects. A similar remark applies for Eqs.(178) and (83) at pN regime. It is mentioned here that only relativistic effects are taken into account at $\mathrm{pN}$ regime. We have found that the results of dissipative case reduce to nondissipative if heat flow vanishes.

\section{References}

[1] Chandrasekhar, S.: Astrophys. J. 140(1964)417.

[2] Cocke, W. J.: Ann. Inst. Henri Poincare A 2(1965)283.

[3] Fowler, W. A.: Astrophys. J. 144(1966)180.

[4] Thorne, K. and Campolattro, A.: Astrophys. J. 149(1967)591.

[5] Herrera, L., Santos, N.O. and Le Denmat, G.: Mon. Not. R. Astron. Soc. 237(1989)257.

[6] Chan, R., Herrera, L. and Santos, N.O.: Mon. Not. R. Astron. Soc. 265(1993)533.

[7] Chan, R., Herrera, L. and Santos, N.O.: Mon. Not. R. Astron. Soc. $267(1994) 637$.

[8] Chan, R.: Mon. Not. R. Astron. Soc. 316(2000)588.

[9] Dev, K. and Gleiser, M.: Gen. Relativ. Gravit. 35(2003)1435.

[10] Sharif, M. and Kausar, H.R.: JCAP 07(2011)022.

[11] Rosseland, S.: Mon. Not. R. Astron. Soc. 84(1924)720.

[12] Bekenstein, J.: Phys. Rev. D 4(1971)2185.

[13] Glazer, I.: Ann. Phys. 101(1976)594.

[14] Zhang, J.L., Chau, W.Y. and Deng, T.Y.: Astrophys. Space Sci. 88(1982)81. 
[15] Thirukkanesh, S. and Maharaj, S.D.: Class. Quantum Grav. $\mathbf{2 5}(2008) 235001$.

[16] Esculpi, M. and Aloma, E.: Eur. Phys. J. C 67(2010)521.

[17] Ernesto, F.E. and Simeone, C.: Phys. Rev. D 83(2011)104009.

[18] Joshi, P.S. and Malafarina, D.: Phys. Rev. D 83(2011)024009.

[19] Sharif, M. and Azam, M.: Effects of Electromagnetic Field on the Dynamical Instability of Expansionfree Gravitational Collapse, Gen. Relativ. Gravit. (2012, to appear); DOI. 10.1007/s10714-012-1333-8.

[20] Sharif, M. and Ahmad, Z.: Gen. Relativ. Gravit. 39(2007)1331.

[21] Di Prisco, A., Herrera, L., MacCallum, M.A.H. and Santos, N.O.: Phys. Rev. D 80(2009)064031.

[22] Nakao, K., Harada, T., Kurita, Y. and Morisawa, Y.: Prog. Theor. Phys. $\mathbf{1 2 2}(2009) 521$.

[23] Sharif, M. and Abbas, G.: J. Phys. Soc. Jpn. 80(2011)104002.

[24] Darmois, G.: Memorial des Sciences Mathematiques (Gautheir-Villars, 1927) Fasc. 25.

[25] Sharif, M. and Abbas, G.: Astrophys. Space Sci. 335(2011)515.

[26] Prisco, D.A., Herrera, L., Denmat, G.L., MacCallum, H.A.M. and Santos, N.O.: Phys. Rev. D76(2007)064017.

[27] Thorne, K.S.: Phys. Rev. B 138(1965)251.

[28] Poisson, E.: A Relaivistic's Toolkit (Cambridge University Press, 2004)

[29] Chao-Guang, H.: Acta Phys. Sin. 4(1995)617.

[30] Berti, E., Cardoso, V. and Starinets, A.O.: Class. Quantum Grav. 26(2009)163001. 